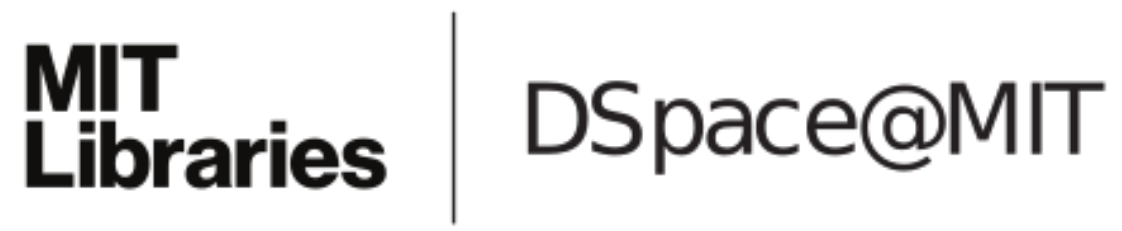

\author{
MIT Open Access Articles
}

Discriminating quantum-optical beam-splitter channels with number-diagonal signal states: Applications to quantum reading and target detection

The MIT Faculty has made this article openly available. Please share how this access benefits you. Your story matters.

Citation: Nair, Ranjith. “Discriminating Quantum-optical Beam-splitter Channels with Numberdiagonal Signal States: Applications to Quantum Reading and Target Detection." Physical Review A 84.3 (2011): [12 pages]. (C2011 American Physical Society.

As Published: http://dx.doi.org/10.1103/PhysRevA.84.032312

Publisher: American Physical Society

Persistent URL: http://hdl.handle.net/1721.1/69245

Version: Final published version: final published article, as it appeared in a journal, conference proceedings, or other formally published context

Terms of Use: Article is made available in accordance with the publisher's policy and may be subject to US copyright law. Please refer to the publisher's site for terms of use. 


\title{
Discriminating quantum-optical beam-splitter channels with number-diagonal signal states: Applications to quantum reading and target detection
}

\author{
Ranjith Nair* \\ Research Laboratory of Electronics, Massachusetts Institute of Technology, Cambridge, Massachusetts 02139, USA
}

(Received 27 May 2011; published 8 September 2011)

\begin{abstract}
We consider the problem of distinguishing, with minimum probability of error, two optical beam-splitter channels with unequal complex-valued reflectivities using general quantum probe states entangled over $M$ signal and $M^{\prime}$ idler mode pairs of which the signal modes are bounced off the beam splitter while the idler modes are retained losslessly. We obtain a lower bound on the output state fidelity valid for any pure input state. We define number-diagonal signal (NDS) states to be input states whose density operator in the signal modes is diagonal in the multimode number basis. For such input states, we derive series formulas for the optimal error probability, the output state fidelity, and the Chernoff-type upper bounds on the error probability. For the special cases of quantum reading of a classical digital memory and target detection (for which the reflectivities are real valued), we show that for a given input signal photon probability distribution, the fidelity is minimized by the NDS states with that distribution and that for a given average total signal energy $N_{s}$, the fidelity is minimized by any multimode Fock state with $N_{s}$ total signal photons. For reading of an ideal memory, it is shown that Fock state inputs minimize the Chernoff bound. For target detection under high-loss conditions, a no-go result showing the lack of appreciable quantum advantage over coherent state transmitters is derived. A comparison of the error probability performance for quantum reading of number state and two-mode squeezed vacuum state (or EPR state) transmitters relative to coherent state transmitters is presented for various values of the reflectances. While the nonclassical states in general perform better than the coherent state, the quantitative performance gains differ depending on the values of the reflectances. The experimental outlook for realizing nonclassical gains from number state transmitters with current technology at moderate to high values of the reflectances is argued to be good.
\end{abstract}

DOI: $10.1103 /$ PhysRevA.84.032312

PACS number(s): 03.67.Hk, 42.50.Ex

\section{INTRODUCTION}

Consider, for $b \in\{0,1\}$, an optical beam-splitter channel taking an input signal mode annihilation operator $\hat{a}_{\text {in }}$ to the output mode annihilation operator $\hat{a}_{\text {out }}$ in the manner of Fig. 1 under the mode transformation:

$$
\left(\begin{array}{l}
\hat{a}_{\text {out }} \\
\hat{e}_{\text {out }}
\end{array}\right)=\left(\begin{array}{cc}
r_{b} e^{i \theta_{b}} & t_{b} \\
t_{b} e^{i \theta_{b}} & -r_{b}
\end{array}\right)\left(\begin{array}{l}
\hat{a}_{\text {in }} \\
\hat{e}_{\text {in }}
\end{array}\right) .
$$

Here, $r_{b}=\sqrt{R_{b}}$ and $t_{b}=\sqrt{T_{b}}$ are real and non-negative field reflectivities with $R_{b}$ and $T_{b}$ the corresponding reflectances and transmittances with $R_{b}+T_{b}=1$. We will assume $R_{0} \leqslant R_{1}$ throughout the paper. We have included, for later purposes, the input and output annihilation operators $\hat{e}_{\text {in }}$ and $\hat{e}_{\text {out }}$ of the other (environment) mode incident at the beam splitter, whose input is assumed to be in the vacuum state. Denoting the $b$-dependent quantum channels induced on the signal mode by the above beam-splitter operation by $\mathcal{E}_{b}$, and assuming that each of these channels has equal a priori probability, we consider the following general strategy to discriminate these two channels with minimum error probability illustrated in Fig. 1: A quantum state source $S$ prepares a pure state $|\psi\rangle$ on the system consisting of $M$ "signal" optical modes $\left\{\hat{a}_{\text {in }}^{m}\right\}_{m=1}^{M}$ and $M^{\prime}$ “idler" modes $\left\{\hat{b}_{\text {in }}^{m^{\prime}}\right\}_{m^{\prime}=1}^{M^{\prime}}$, allowing for any entanglement across the signal modes and between the signal and idler modes. The $M$ signal modes are passed through $\mathcal{E}_{b}$

*rnair@mit.edu with the idler modes unchanged, giving rise, in general, to mixed density operators

$$
\rho_{b}=\mathcal{E}_{b}^{\otimes^{M}} \otimes \text { id }^{\otimes^{M^{\prime}}}(|\psi\rangle\langle\psi|)
$$

on the signal-idler Hilbert space depending on the value of $b$, where id denotes the identity channel on the idler modes. This joint state is now measured at the detector $D$ using the Helstrom quantum measurement that yields minimum error probability in distinguishing the two states [1]. In view of the fact that loss is ubiquitous in quantum state transmission, processing, and detection, and because of the well-known sensitivity of nonclassical states to loss, we will, in general, assume that $r_{b}<1$. On the other hand, at room temperature and at optical wavelengths $\sim 1 \mu \mathrm{m}$, the average number of thermal noise photons per space-time mode is ideally around $10^{-21}$, allowing us realistically to neglect it, although it is known that interesting nonclassical gains are obtained in high-loss high-noise conditions such as those in "quantum illumination" [2].

Many interesting problems are encompassed by the above model. The case of $r_{0}=0$ (and possibly $r_{1} \ll 1$ ) corresponds to a target detection scenario (identical to quantum illumination [2] but for the absence of thermal noise) in which $b=0$ (1) represents the absence (presence) of a reflecting target of effective field reflectivity $r_{1}$. The more general case of nonzero $r_{0}$ but $\Delta \equiv \theta_{1}-\theta_{0}=0$ corresponds to the recently proposed quantum reading of a classical digital memory [3]. The case of $r_{0}=r_{1}<1, \Delta \neq 0$ models the lossy discrimination of channels differing only in phase shift (or path length) and 


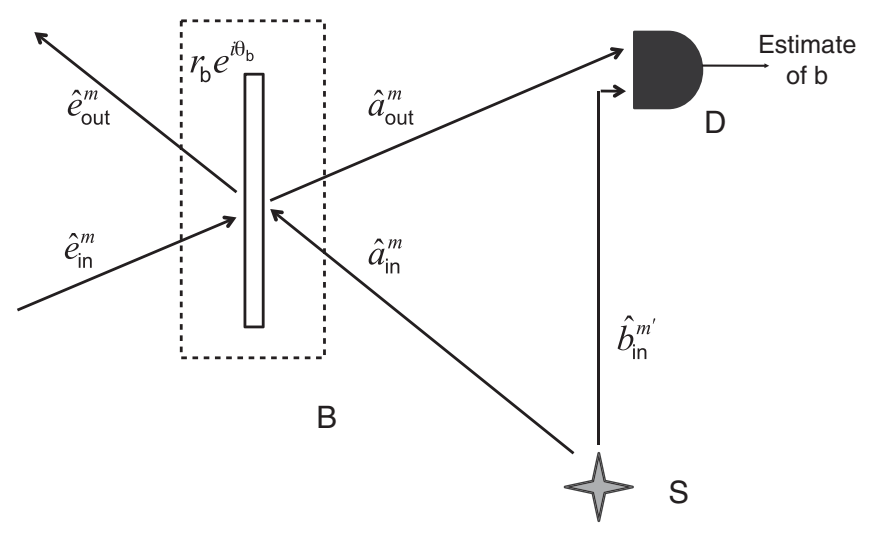

FIG. 1. Schematic of setup for determining which of two beamsplitter channels (indexed by $b$ ) of the form of Eq. (1) is present within a black box B. A quantum state source $S$ produces a pure state of $M$ signal modes $\left\{\hat{a}_{\text {in }}^{m}\right\}_{m=1}^{M}$ and $M^{\prime}$ idler modes $\left\{\hat{b}_{\text {in }}^{m^{\prime}}\right\}_{m^{\prime}=1}^{M^{\prime}}$ of which one signal-idler pair is shown. Each signal mode reflects off the beam splitter while the idler mode is unaffected. The environment modes $\left\{\hat{e}_{\mathrm{in}}^{m}\right\}_{m=1}^{M}$ coupling to the beam splitter are all in the vacuum state. The detection module $D$ performs the minimum error probability (Helstrom) measurement for distinguishing the two possible $(M+$ $M^{\prime}$ )-mode quantum states corresponding to $b=0$ and $b=1$.

is related to the problem of estimation of a continuous optical phase parameter.

Given this background, the following question may now be posed: Among all $|\psi\rangle$ 's with average total energy $N_{s}$ in the signal modes, which state minimizes the average error probability in distinguishing the channels, and how much improvement over a classical state of the same energy is attainable? A multimode classical state is a state with a non-negative $P$ representation $[4,5]$. Thus, $\rho$ given by

$$
\rho=\int P(\boldsymbol{\alpha})|\boldsymbol{\alpha}\rangle\langle\boldsymbol{\alpha}| d^{2} \boldsymbol{\alpha}
$$

is classical if $P(\boldsymbol{\alpha}) \geqslant 0$ for $|\boldsymbol{\alpha}\rangle$, an $M$-mode coherent state, so $\rho$ is a probabilistic mixture of product coherent states. Comparison of the performance of a proposed input state to this class of states is important since, as their definition implies, the latter are readily prepared by modulating laser fields with classical random numbers. In particular, we will compare performance with the pure coherent state $\left|\sqrt{N_{s}}\right\rangle \otimes|0\rangle^{\otimes^{M-1}}[6]$.

A related version of the general problem of discriminating two beam-splitter channels has been addressed recently in Ref. [7]. The scenario of Ref. [7] differs from ours in that it is assumed, referring to Fig. 1, that the combined state of $\hat{e}_{\text {in }}$ and $\hat{a}_{\text {in }}$ may be chosen subject to a total energy constraint and that both the beam-splitter output modes $\hat{a}_{\text {out }}$ and $\hat{e}_{\text {out }}$ are available for a Helstrom detector to measure, in addition to the idler mode $\hat{b}_{\text {in }}$. As such, the problem of Ref. [7] is one of discrimating two unitary transformations as opposed to the nonunitary channel discrimination problem considered here. Nevertheless, it is clear that, for $M=1$, the minimum error probability attainable at given $N_{s}$ in the setting of Ref. [7] is not greater than that for our more restrictive setting. On the other hand, the case of $M>1$ was not considered in Ref. [7]. We note that the problem in the form addressed here is of more relevance to long-range scenarios where the black box $B$ of
Fig. 1, and, therefore, the input and output environment modes, are not directly accessible to the user who controls the state source $S$ and the detector $D$. It also models quantum reading scenarios where the memory itself (e.g., a CD or DVD) need not be modified in a major way. When such modifications to the memory are contemplated, the scenario of Ref. [7] becomes interesting.

This paper provides several partial answers to the broad question posed above. In Sec. II, we derive a lower bound on the Jozsa-Uhlmann fidelity $[8,9]$ between $\rho_{0}$ and $\rho_{1}$

$$
\mathcal{F}=\operatorname{Tr}^{2} \sqrt{\sqrt{\rho_{0}} \rho_{1} \sqrt{\rho_{0}}}
$$

that is valid for any input state $|\psi\rangle$ [10]. This further yields a lower bound on the minimum error probability of distinguishing the output states. In Sec. III, we specialize to input states whose reduced density operator on the signal modes is a mixture of multimode number states; in this paper such states are referred to as number-diagonal signal (NDS) states. It was shown recently in Ref. [11] that NDS states are optimum input states according to many possible performance criteria for a large class of image sensing problems of which the minimum error probability discrimination between two beam-splitter channels that is the subject of the present paper is a special case. We mention that, in the contexts of communication and key distribution between two users, a class of states related to the NDS states was introduced in Ref. [12] and referred to as photon-number entangled states (PNES). A PNES is a pure state of a single signal-idler mode pair that is diagonal in the number state basis of both the signal and idler modes. Since the states we consider do not have to be number state diagonal in the idler modes, we believe the term number-diagonal signal state is more appropriate. However, it is the case that a given single-mode NDS state can be related to a corresponding PNES by a unitary transformation on the idler modes without changing the error performance.

The NDS states include, but are not limited to, number states, the two-mode squeezed vacuum (or EPR) states used in Refs. [2,3], the NOON states [13], the related $\left|m:: m^{\prime}\right\rangle$ states of Ref. [14], the pair coherent states (PCS) [15], and several other states studied in the context of phase estimation [16]. For this class of states, we show that $\rho_{0}$ and $\rho_{1}$ of Eq. (2) are easily diagonalized. The minimum possible error probability of discriminating any two states (with equal a priori probabilities) is given by the Helstrom formula [1]

$$
\bar{P}_{e}=\frac{1}{2}-\frac{1}{4}\left\|\rho_{0}-\rho_{1}\right\|_{1},
$$

where $\|A\|_{1}$ is the trace norm which equals, for self-adjoint $A$, the sum of the absolute values of the eigenvalues of $A$. For noncommuting mixed states, $\bar{P}_{e}$ is notoriously hard to calculate. For input NDS states, however, we show in Sec. III that the eigenvectors of $\rho_{0}$ and $\rho_{1}$ have a mutual inner product structure that permits the calculation of $\bar{P}_{e}$ as an, in general, infinite series. The fidelity (4) also yields the following upper and lower bounds on $\bar{P}_{e}$ [17] (the first inequality follows from a binomial expansion of its right-hand side):

$$
\frac{1}{4} \mathcal{F} \leqslant \frac{1}{2}(1-\sqrt{1-\mathcal{F}}) \leqslant \bar{P}_{e} \leqslant \frac{1}{2} \sqrt{\mathcal{F}} .
$$


Also of interest are the "Chernoff-type" upper bounds:

$$
\bar{P}_{e} \leqslant \frac{1}{2} Q(s), \quad s \in[0,1],
$$

where $Q(s)=\operatorname{Tr}\left[\rho_{0}^{s} \rho_{1}^{1-s}\right]$ and the Chernoff bound $Q$ [18] is the best such bound:

$$
\bar{P}_{e} \leqslant \frac{1}{2} Q
$$

where

$$
Q=\min _{s \in[0,1]} Q(s) .
$$

The Bhattacharyya bound is (7) with $s=1 / 2$. This terminology was introduced in Ref. [19] and the bound was applied for the first time in Ref. [2]. We show here that, for NDS input states, $\mathcal{F}$ and $Q(s)$ are also calculable as infinite series in general and have closed-form expressions in some cases.

In Sec. IV, we apply these results to quantum reading and target detection, i.e., to the cases where $\Delta=0$. The input state consisting of $M$ signal and idler mode pairs from the output of a parametric down-conversion process was shown in Ref. [3] to yield, in some regions of $M$ and $N_{s}$, surprisingly better error probability in quantum reading than that obtainable from any classical state of the same energy [20]. In this paper, we first show in Sec. IVA that, for any given signal photon number distribution, the general fidelity lower bound derived in Sec. I is attained by NDS states. Further, the NDS states minimizing the fidelity for a given $N_{s}$ and $M$ are the multimode Fock states of total photon number $N_{s}$. In Sec. IVA1, we lower bound the error probability for quantum reading and target detection via the fidelity for general input states. In Sec. IVA2, for the case of $R_{1}=1$, we show that the Fock states give the lowest Chernoff bound among all pure input states of given energy. In Sec. IVA3, we state a fidelity-based error probability lower bound for target detection with a general input state. In the limit $R_{1} \ll 1$ of large loss, we derive a no-go result that rules out appreciable quantum advantage over coherent states.

In Secs. IVB- IVD, we characterize in detail the error probability performance of coherent states, number states, and the EPR states. We show that the performance of all the multimode Fock states of total photon number $N_{s}$ are identical, a fact that helps practical implementation. For EPR states, we show that the application of the results of Sec. III yields the same analytical results as the methods used in Ref. [3]. In Sec. IVE1, we compare the quantitative performance of coherent, number, and EPR states for some typical reflectance values and demonstrate nonclassical gains. In Sec. IVE2, we consider the technological feasibility of achieving the nonclassical gains in quantum reading.

\section{LOWER BOUND ON OUTPUT STATE FIDELITY}

Consider an arbitrary $\left(M^{\prime}+M\right)$-mode state $|\psi\rangle$ in the photon number representation

$$
|\psi\rangle=\sum_{\mathbf{m}, \mathbf{n}} c_{\mathbf{m}, \mathbf{n}}|\mathbf{m}\rangle_{I}|\mathbf{n}\rangle_{S},
$$

where $|\mathbf{m}\rangle_{I}=\left|m_{1}\right\rangle_{I_{1}} \otimes \cdots \otimes\left|m_{M^{\prime}}\right\rangle_{I_{M^{\prime}}}$ and $|\mathbf{n}\rangle_{S}=\left|n_{1}\right\rangle_{S_{1}} \otimes$ $\cdots \otimes\left|n_{M}\right\rangle_{S_{M}}$ are, respectively, the photon number states of the $M$-mode ( $M^{\prime}$-mode) signal (idler). After augmenting the above state with the vacuum $|\mathbf{0}\rangle_{E}$ of the $M$ environment modes $\left\{\hat{e}_{\text {in }}\right\}_{m=1}^{M}$ of (1), we may write the Schrödinger-picture evolution corresponding to the Heisenberg-picture evolution (1) of the joint system to one of two pure states as

$$
\left|\psi^{(b)}\right\rangle=\sum_{\mathbf{m}, \mathbf{n}} c_{\mathbf{m}, \mathbf{n}} \sum_{\mathbf{k} \leqslant \mathbf{n}} A_{\mathbf{n} ; \mathbf{k}}^{(b)}|\mathbf{m}\rangle_{I}|\mathbf{n}-\mathbf{k}\rangle_{S}|\mathbf{k}\rangle_{E}
$$

where the amplitude $A_{\mathbf{n} ; \mathbf{k}}^{(b)}$ is given by

$$
A_{\mathbf{n} ; \mathbf{k}}^{(b)}=\prod_{m=1}^{M}\left[\sqrt{\left(\begin{array}{l}
n_{m} \\
k_{m}
\end{array}\right)} e^{i n_{m} \theta_{\mathrm{b}}} r_{b}^{n_{m}-k_{m}} t_{b}^{k_{m}}\right] .
$$

In Eq. (11), $\mathbf{k} \leqslant \mathbf{n}$ is to be understood as a component-wise inequality. We then have $\rho_{b}=\operatorname{Tr}_{E}\left[\left|\psi^{(b)}\right\rangle\left\langle\psi^{(b)}\right|\right]$ so the $\left|\psi^{(b)}\right\rangle$ are purifications [21] of the respective $\rho_{b}$. From Eq. (11), it follows that the overlap $O=\left|\left\langle\psi^{(0)} \mid \psi^{(1)}\right\rangle\right|^{2}$ equals

$O$

$$
\begin{aligned}
& =\left.\left.\left|\sum_{\mathbf{m}, \mathbf{n}}\right| c_{\mathbf{m}, \mathbf{n}}\right|^{2} \sum_{\mathbf{k} \leqslant \mathbf{n}} \prod_{m=1}^{M}\left[\left(\begin{array}{l}
n_{m} \\
k_{m}
\end{array}\right) e^{i n_{m} \Delta}\left(r_{0} r_{1}\right)^{n_{m}-k_{m}}\left(t_{0} t_{1}\right)^{k_{m}}\right]\right|^{2} \\
& =\left|\sum_{\mathbf{n}} p_{\mathbf{n}} \sum_{\mathbf{k} \leqslant \mathbf{n}} \prod_{m=1}^{M}\left[\left(\begin{array}{l}
n_{m} \\
k_{m}
\end{array}\right) e^{i n_{m} \Delta}\left(r_{0} r_{1}\right)^{n_{m}-k_{m}}\left(t_{0} t_{1}\right)^{k_{m}}\right]\right|^{2} \\
& =\left|\sum_{\mathbf{n}} p_{\mathbf{n}} \prod_{m=1}^{M}\left[e^{i n_{m} \Delta}\left(r_{0} r_{1}+t_{0} t_{1}\right)^{n_{m}}\right]\right|^{2} \\
& =\left|\sum_{n=0}^{\infty} p_{n} e^{i n \Delta}\left(r_{0} r_{1}+t_{0} t_{1}\right)^{n}\right|^{2}
\end{aligned}
$$

where $p_{\mathbf{n}}=\sum_{\mathbf{m}}\left|c_{\mathbf{m}, \mathbf{n}}\right|^{2}$ is the multimode photon probability distribution in the signal modes. In going from Eq. (16) to Eq. (17), we have reordered the sum of Eq. (16) over the single index $n=\sum_{m=1}^{M} n_{m}$, with $p_{n}$ being the probability distribution of the total signal photon number

$$
p_{n}=\sum_{\mathbf{n}: \sum_{m=1}^{M} n_{m}=n} p_{\mathbf{n}}
$$

Uhlmann's theorem $[8,9,21]$ states that the fidelity $\mathcal{F}$ is the maximum overlap over all purifications of $\rho_{0}$ and $\rho_{1}$, so we have the lower bound

$$
O \leqslant \mathcal{F}
$$

for the $O$ of Eq. (17). For any proposed input state $|\psi\rangle$, the overlap $O(|\psi\rangle)$ can be calculated via Eq. (17) in terms of the signal photon probability distribution of $|\psi\rangle$. Application of the inequalities (19) and (6) yields the following lower bound on $\bar{P}_{e}$ :

$$
\frac{1}{2}(1-\sqrt{1-O(|\psi\rangle)}) \leqslant \bar{P}_{e}[|\psi\rangle] .
$$

The cases of quantum reading and target detection for which $\Delta=0$ are further developed in Sec. IVA. 


\section{NDS STATES: MINIMUM ERROR PROBABILITY, OUTPUT STATE FIDELITY, AND CHERNOFF-TYPE BOUNDS}

We define NDS states to be states whose reduced density operator in the $M$ signal modes is diagonal in the product number (Fock) state basis. A pure NDS state then has the representation

$$
|\psi\rangle=\sum_{\mathbf{n}} c_{\mathbf{n}}\left|\phi_{\mathbf{n}}\right\rangle_{I}|\mathbf{n}\rangle_{S},
$$

where $\left|\phi_{\mathbf{n}}\right\rangle_{I}$ is any orthonormal set of states on the idler modes; the above equation is essentially a Schmidt decomposition [21] of $|\psi\rangle$. This is a wide class of states, many of which have been intensively studied in quantum optics and have interesting applications in quantum information and metrology [2,3,12-16].

We now show that, for NDS inputs, $\rho_{0}$ and $\rho_{1}$ have a form helpful for calculations. After propagation through the beamsplitter channel we obtain, as in Eq. (11), the purifications

$$
\begin{aligned}
\left|\psi^{(\mathrm{b})}\right\rangle & =\sum_{\mathbf{n}} c_{\mathbf{n}} \sum_{\mathbf{k} \leqslant \mathbf{n}} A_{\mathbf{n} ; \mathbf{k}}^{(\mathrm{b})}\left|\phi_{\mathbf{n}}\right\rangle_{I}|\mathbf{n}-\mathbf{k}\rangle_{S}|\mathbf{k}\rangle_{E} \\
& =\sum_{\mathbf{k}}\left(\sum_{\mathbf{n}: \mathbf{n} \geqslant \mathbf{k}} c_{\mathbf{n}} A_{\mathbf{n} ; \mathbf{k}}^{(\mathbf{b})}\left|\phi_{\mathbf{n}}\right\rangle_{I}|\mathbf{n}-\mathbf{k}\rangle_{S}\right)|\mathbf{k}\rangle_{E} \\
& \equiv \sum_{\mathbf{k}}\left|\psi_{\mathbf{k}}^{(\mathbf{b})}\right\rangle|\mathbf{k}\rangle_{E},
\end{aligned}
$$

where

$$
\left|\psi_{\mathbf{k}}^{(\mathbf{b})}\right\rangle=\sum_{\mathbf{n}: \mathbf{n} \geqslant \mathbf{k}} c_{\mathbf{n}} A_{\mathbf{n} ; \mathbf{k}}^{(\mathbf{b})}\left|\phi_{\mathbf{n}}\right\rangle_{I}|\mathbf{n}-\mathbf{k}\rangle_{S}
$$

are un-normalized joint signal-idler states. Since the $\left\{\left|\phi_{\mathbf{n}}\right\rangle_{I}\right\}$ are an orthonormal set by the NDS state assumption, we see that the orthogonality relations

$$
\left\langle\psi_{\mathbf{k}}^{(b)} \mid \psi_{\mathbf{k}^{\prime}}^{(b)}\right\rangle=p_{\mathbf{k}}^{(b)} \delta_{\mathbf{k}, \mathbf{k}^{\prime}}
$$

hold because the $\left|\psi_{\mathbf{k}}^{(b)}\right\rangle$ and $\left|\psi_{\mathbf{k}^{\prime}}^{(b)}\right\rangle$ of Eq. (25) are termwise orthogonal when $\mathbf{k} \neq \mathbf{k}^{\prime}$. Here $p_{\mathbf{k}}^{(b)}$ is the probability, conditioned on $b$, of finding $\mathbf{k}$ photons in the output environment modes and is given by

$$
\begin{aligned}
p_{\mathbf{k}}^{(b)} & =\sum_{\mathbf{n}: \mathbf{n} \geqslant \mathbf{k}}\left|c_{\mathbf{n}} A_{\mathbf{n}: \mathbf{k}}^{(b)}\right|^{2} \equiv \sum_{\mathbf{n}: \mathbf{n} \geqslant \mathbf{k}} p_{\mathbf{n}}\left|A_{\mathbf{n} ; \mathbf{k}}^{(b)}\right|^{2} \\
& =\sum_{\mathbf{n}: \mathbf{n} \geqslant \mathbf{k}} p_{\mathbf{n}} \prod_{m=1}^{M}\left[\left(\begin{array}{c}
n_{m} \\
k_{m}
\end{array}\right) R_{\mathrm{b}}^{\left(n_{m}-k_{m}\right)} T_{\mathrm{b}}^{k_{m}}\right] .
\end{aligned}
$$

Again by the NDS state assumption, we have

$$
\left\langle\psi_{\mathbf{k}}^{(0)} \mid \psi_{\mathbf{k}^{\prime}}^{(1)}\right\rangle=I_{\mathbf{k}} \delta_{\mathbf{k}, \mathbf{k}^{\prime}}
$$

for

$$
\begin{aligned}
I_{\mathbf{k}} & =\sum_{\mathbf{n}: \mathbf{n} \geqslant \mathbf{k}}\left|c_{\mathbf{n}}\right|^{2}\left(A_{\mathbf{n} ; \mathbf{k}}^{(0)}\right)^{*} A_{\mathbf{n} ; \mathbf{k}}^{(1)} \\
& =\sum_{\mathbf{n}: \mathbf{n} \geqslant \mathbf{k}} p_{\mathbf{n}} \prod_{m=1}^{M}\left[\left(\begin{array}{l}
n_{m} \\
k_{m}
\end{array}\right) e^{i n_{m} \Delta}\left(r_{0} r_{1}\right)^{n_{m}-k_{m}}\left(t_{0} t_{1}\right)^{k_{m}}\right] .
\end{aligned}
$$

The orthogonality relations (26) and (29) are key to the mathematical tractability of the optimal error probability problem for NDS input states.

\section{A. Optimal (Helstrom) detection: Error probability and measurement operators}

Tracing over the environment modes in Eq. (24), we obtain $\rho_{0}$ and $\rho_{1}$, which are already in diagonal form by virtue of (26):

$$
\rho_{b}=\sum_{\mathbf{k}}\left|\psi_{\mathbf{k}}^{(b)}\right\rangle\left\langle\psi_{\mathbf{k}}^{(b)}\right| .
$$

For $\mathcal{H}_{\mathbf{k}}=\operatorname{span}\left\{\psi_{\mathbf{k}}^{(0)}, \psi_{\mathbf{k}}^{(1)}\right\}$, we have, via Eqs. (26) and (29), that the two-dimensional spaces $\left\{\mathcal{H}_{\mathbf{k}}\right\}$ are mutually orthogonal. The joint signal-idler Hilbert space $\mathcal{H}$ may then be expressed as an orthogonal direct sum of the $\left\{\mathcal{H}_{\mathbf{k}}\right\}$ with an additional component $\mathcal{H}_{\perp}$ orthogonal to all the $\mathcal{H}_{\mathbf{k}}$ :

$$
\mathcal{H}=\bigoplus_{\mathbf{k}} \mathcal{H}_{\mathbf{k}} \oplus \mathcal{H}_{\perp}
$$

For the purpose of distinguishing $\rho_{0}$ and $\rho_{1}$, we may restrict the domain of definition of the difference density operator $\Delta \rho \equiv \rho_{0}-\rho_{1}$ appearing in the Helstrom formula (5) to $\bigoplus_{\mathbf{k}} \mathcal{H}_{\mathbf{k}}$ since the support of both $\rho_{0}$ and $\rho_{1}$ is orthogonal to $\mathcal{H}_{\perp}$. Corresponding to (33), $\Delta \rho$ may be decomposed into a direct sum of operators on $\mathcal{H}_{\mathbf{k}}$

$$
\Delta \rho=\left.\bigoplus_{\mathbf{k}} \Delta \rho\right|_{\mathbf{k}},\left.\quad \Delta \rho\right|_{\mathbf{k}} \in \mathcal{L}\left(\mathcal{H}_{\mathbf{k}}\right),
$$

where

$$
\left.\Delta \rho\right|_{\mathbf{k}}=\left|\psi_{\mathbf{k}}^{(0)}\right\rangle\left\langle\psi_{\mathbf{k}}^{(0)}|-| \psi_{\mathbf{k}}^{(1)}\right\rangle\left\langle\psi_{\mathbf{k}}^{(1)}\right| .
$$

Performing a Gram-Schmidt orthonormalization on each $\mathcal{H}_{\mathbf{k}}=\operatorname{span}\left\{\psi_{\mathbf{k}}^{(0)}, \psi_{\mathbf{k}}^{(1)}\right\}$ with $\psi_{\mathbf{k}}^{(0)} /\left\|\psi_{\mathbf{k}}^{(0)}\right\|$ as the first basis vector, we may write the $2 \times 2$ matrix of $\left.\Delta \rho\right|_{\mathbf{k}}$ in the GramSchmidt basis as [22]:

$\left.\Delta \rho\right|_{\mathbf{k}}=\left(\begin{array}{lc}p_{\mathbf{k}}^{(0)}-\left|I_{\mathbf{k}}\right|^{2} / p_{\mathbf{k}}^{(0)} & -\frac{I_{\mathbf{k}}}{p_{\mathbf{k}}^{(0)}} \sqrt{p_{\mathbf{k}}^{(0)} p_{\mathbf{k}}^{(1)}-\left|I_{\mathbf{k}}\right|^{2}} \\ -\frac{I_{\mathbf{k}}^{*}}{p_{\mathbf{k}}^{(0)}} \sqrt{p_{\mathbf{k}}^{(0)} p_{\mathbf{k}}^{(1)}-\left|I_{\mathbf{k}}\right|^{2}} & -p_{\mathbf{k}}^{(1)}+\left|I_{\mathbf{k}}\right|^{2} / p_{\mathbf{k}}^{(0)}\end{array}\right)$.

On calculating the eigenvalues and trace norm of the above matrix, the minimum error probability follows as

$$
\begin{aligned}
\bar{P}_{e} & =\frac{1}{2}-\frac{1}{4}\left\|\bigoplus_{\mathbf{k}}\left(\left.\Delta \rho\right|_{\mathbf{k}}\right)\right\|_{1} \\
& =\frac{1}{2}-\frac{1}{4} \sum_{\mathbf{k}}\left\|\left.\Delta \rho\right|_{\mathbf{k}}\right\|_{1} \\
& =\frac{1}{2}-\frac{1}{4} \sum_{\mathbf{k}}\left[\left(p_{\mathbf{k}}^{(0)}+p_{\mathbf{k}}^{(1)}\right)^{2}-4\left|I_{\mathbf{k}}\right|^{2}\right]^{1 / 2} .
\end{aligned}
$$

Although the above sum may be hard to evaluate analytically, numerical computation to any desired accuracy is always possible. 
The discussion above also yields the abstract mathematical description of the measurement operators for optimally discriminating $\rho_{0}$ and $\rho_{1}$. The optimal measurement consists of two orthogonal projection operators $\Pi_{0}$ and $\Pi_{1}$ augmented with an additional projection operator $\Pi_{\perp}$ onto $\mathcal{H}_{\perp}$ to make a complete projective measurement with $\Pi_{0}+\Pi_{1}+\Pi_{\perp}=I_{\mathrm{IS}}$, the identity on $\mathcal{H}$. Note that the state never projects onto $\mathcal{H}_{\perp}$. $\Pi_{0}$ and $\Pi_{1}$ are given in the usual way [1] by

$$
\Pi_{0}=\sum_{\mathbf{k}} \Pi_{+}\left\{\left.\Delta \rho\right|_{\mathbf{k}}\right\}
$$

and

$$
\Pi_{1}=\sum_{\mathbf{k}} \Pi_{-}\left\{\left.\Delta \rho\right|_{\mathbf{k}}\right\}
$$

where $\Pi_{+}\left(\left.\Delta \rho\right|_{\mathbf{k}}\right)$ and $\Pi_{+}\left(\left.\Delta \rho\right|_{\mathbf{k}}\right)$ are respectively the projection operators onto the one-dimensional eigenspaces corresponding to the positive and negative eigenvalues of $\left.\Delta \rho\right|_{\mathbf{k}}$. In somewhat more physical language, we may view the optimum measurement as a two-stage measurement. In the first stage, we perform a quantum nondemolition (QND) measurement, i.e., a measurement that projects the state onto one of several orthogonal Hilbert spaces without destroying it. In our case, the received state is projected into one of the orthogonal spaces $\mathcal{H}_{\mathbf{k}}$. Depending on the value of $\mathbf{k}$, we further make on $\mathcal{H}_{\mathbf{k}}$ the binary Helstrom measurement corresponding to the optimum discrimination of $\left|\psi_{\mathbf{k}}^{(0)}\right\rangle$ and $\left|\psi_{\mathbf{k}}^{(1)}\right\rangle$ with conditional probabilities $p_{\mathbf{k}}^{(0)} /\left(p_{\mathbf{k}}^{(0)}+p_{\mathbf{k}}^{(1)}\right)$ and $p_{\mathbf{k}}^{(1)} /\left(p_{\mathbf{k}}^{(0)}+p_{\mathbf{k}}^{(1)}\right)$, respectively. From this description, we may expect that this measurement is hard to realize in the laboratory since the eigenvectors of $\left.\Delta \rho\right|_{\mathbf{k}}$ are $\left(M^{\prime}+M\right)$-mode entangled states in general.

The case of unequal a priori probabilities, say $\pi_{0}$ and $\pi_{1}$ for $\rho_{0}$ and $\rho_{1}$, may be handled similarly. $\bar{P}_{e}$ is now given by

$$
\bar{P}_{e}=\frac{1}{2}-\frac{1}{2}\left\|\pi_{0} \rho_{0}-\pi_{1} \rho_{1}\right\|_{1} .
$$

The operator $\pi_{0} \rho_{0}-\pi_{1} \rho_{1}$ also has an orthogonal direct sum decomposition over the $\left\{\mathcal{H}_{\mathbf{k}}\right\}$ (which are unchanged). However, the matrices for $\left.\Delta \rho\right|_{\mathbf{k}}$ differ from Eq. (36) and include the prior probabilities. Diagonalizing these matrices yields the optimal error probability and the measurement operators.

\section{B. Fidelity and Chernoff-type bounds}

The optimum error probability $\bar{P}_{e}$ is usefully bounded by the fidelity and Chernoff-type bounds (6) and (7). For NDS states, it is straightforward to show, using Eqs. (26) and (29) in the definition (4), that the fidelity is given by

$$
\begin{gathered}
\mathcal{F}=\left(\sum_{\mathbf{k}}\left|I_{\mathbf{k}}\right|\right)^{2} \\
=\left[\sum_{\mathbf{k}}\left|\sum_{\mathbf{n}: \mathbf{n} \geqslant \mathbf{k}} p_{\mathbf{n}} \prod_{m=1}^{M}\left(\begin{array}{l}
n_{m} \\
k_{m}
\end{array}\right) e^{i n_{m} \Delta}\left(r_{0} r_{1}\right)^{n_{m}-k_{m}}\left(t_{0} t_{1}\right)^{k_{m}}\right|\right]^{2},
\end{gathered}
$$

where we have used (31). The presence of the absolute value sign prevents simplification of the above expression without further assumptions, but we note that, for $\Delta \neq 0$, Eq. (44) is greater, and hence more informative, than the general lower bound (15).

The Chernoff-type quantities may be likewise computed to equal

$$
Q(s)=\sum_{\mathbf{k}}\left[p_{\mathbf{k}}^{(0)}\right]^{s-1}\left[p_{\mathbf{k}}^{(1)}\right]^{-s}\left|I_{\mathbf{k}}\right|^{2},
$$

for $s \in[0,1]$. The "singularity" in the terms for which $p_{\mathbf{k}}^{(\mathrm{b})}=$ 0 is only apparent as $I_{\mathbf{k}}$ is also zero for those terms. Therefore, we need sum only over terms for which both $p_{\mathbf{k}}^{(\text {b) }} \neq 0$. We see that $Q(0)$ and $Q(1)$ do not equal 1 , since $\left|I_{\mathbf{k}}\right|^{2}<p_{\mathbf{k}}^{(0)} p_{\mathbf{k}}^{(1)}$ in general (see Ref. [22]). This is explained by the fact that the support of $\rho_{0}$, i.e., $\operatorname{span}\left\{\psi_{\mathbf{k}}^{(0)}\right\} \neq \operatorname{span}\left\{\psi_{\mathbf{k}}^{(1)}\right\}$, the support of $\rho_{1}$. Indeed, since $\psi_{\mathbf{k}}^{(0)} \not<\psi_{\mathbf{k}}^{(1)}$ for any $\mathbf{k}, \rho_{0}$ and $\rho_{1}$ do not have the same support on $\mathcal{H}_{\mathbf{k}}$, and, consequently, also on $\oplus_{\mathbf{k}} \mathcal{H}_{\mathbf{k}}$. As a result, $Q(0)=\operatorname{Tr}\left[\rho_{0}^{0} \rho_{1}\right]=\operatorname{Tr}\left[P_{0} \rho_{1}\right] \neq \operatorname{Tr}\left[I \rho_{1}\right]=1$, for $P_{0}$ the projection operator onto the support of $\rho_{0}$ and $I$ the identity operator on $\oplus_{\mathbf{k}} \mathcal{H}_{\mathbf{k}}$. Similarly $\rho_{1}^{0}=P_{1} \neq I$ is the projector onto the support of $\rho_{1}$ and so $Q(1)=\operatorname{Tr}\left[\rho_{0} P_{1}\right] \neq 1$.

For a transmitted state $|\Psi\rangle=\otimes_{m=1}^{M}\left|\psi_{m}\right\rangle$ that is a product of $M$ signal-idler states $\left|\psi_{m}\right\rangle$, we have the multiplicative properties

$$
\mathcal{F}(|\Psi\rangle)=\prod_{m=1}^{M} \mathcal{F}\left(\left|\psi_{m}\right\rangle\right)
$$

and

$$
Q(s)(|\Psi\rangle)=\prod_{m=1}^{M} Q(s)(|\psi\rangle),
$$

which simplify computations by converting the sum over vector $\mathbf{k}$ to a product of scalar sums. We illustrate the results of this subsection in Sec. IVD by applying them to quantum reading with the EPR state.

\section{QUANTUM READING AND TARGET DETECTION}

\section{A. Output state fidelity and related error probability bounds}

For the quantum reading and target detection scenarios, we have $\Delta=\theta_{1}-\theta_{0}=0$. In Sec. II, we obtained the lower bound (19) on the output state fidelity in terms of the input state's signal photon probability distribution $\left\{p_{n}\right\}$. For an NDS input state with that $\left\{p_{n}\right\}$, the fidelity (43) evaluates to

$$
\mathcal{F}=\left[\sum_{n=0}^{\infty} p_{n}\left(r_{0} r_{1}+t_{0} t_{1}\right)^{n}\right]^{2},
$$

which is exactly the general fidelity lower bound [Eqs. (17)(19)] of Sec. II with $\Delta=0$. Thus, among all input states with a given $\left\{p_{n}\right\}$, the NDS states with that $\left\{p_{n}\right\}$ minimize the fidelity.

Further, since $\mu \equiv r_{0} r_{1}+t_{0} t_{1}<1$ when at least one $r_{\mathrm{b}}<1$, we have by the convexity of the function $x \mapsto \mu^{x}$ and Jensen's 
inequality [23] that, for a given total signal energy $N_{s}$,

$$
\begin{aligned}
\mathcal{F} & =\left[\sum_{n=0}^{\infty} p_{n}\left(r_{0} r_{1}+t_{0} t_{1}\right)^{n}\right]^{2} \\
& \geqslant\left[\left(r_{0} r_{1}+t_{0} t_{1}\right)^{\sum_{n=0}^{\infty} n p_{n}}\right]^{2} \\
& =\left(r_{0} r_{1}+t_{0} t_{1}\right)^{2 N_{s}} \\
& \equiv \mathcal{F}_{\min }\left(N_{s}\right) .
\end{aligned}
$$

The inequality (50) is an equality (at least at integer values of $N_{s}$ ) for precisely the states having $N_{s}$ total signal photons with probability 1 . The only NDS states with this property are the multimode Fock states $\left|N_{1}\right\rangle \otimes \cdots \otimes\left|N_{M}\right\rangle$ with total number of photons $\sum_{m=1}^{M} N_{m}=N_{s}$. We have thus shown that a (possibly multimode) Fock state with $N_{s}$ photons minimizes the output fidelity at given $N_{s}$.

The above argument, employing convexity as it does, does not go through when $\Delta \neq 0$. Moreover, even for $\Delta=0$, achieving minimum fidelity is not equivalent to achieving minimum $\bar{P}_{e}$, as will be evident in the examples to follow in Secs. IVC-IVE.

\section{Error probability lower bounds}

Since $\mathcal{F}_{\min }\left(N_{s}\right)$ of (52) is a lower bound on the output state fidelity for any multimode pure input state $|\psi\rangle$ of energy $N_{s}$, we obtain the universal lower bound

$$
\begin{aligned}
\frac{1}{2}\left[1-\sqrt{1-\mathcal{F}_{\min }\left(N_{s}\right)}\right] & =\frac{1}{2}\left[1-\sqrt{1-\left(r_{0} r_{1}+t_{0} t_{1}\right)^{2 N_{s}}}\right] \\
& \leqslant \bar{P}_{e}[|\psi\rangle]
\end{aligned}
$$

on the output error probability $\bar{P}_{e}[|\psi\rangle]$ using (6).

For any input state $|\Psi\rangle$ consisting of $N$ copies of an $(M+$ $M^{\prime}$ )-mode signal-idler state $|\psi\rangle$

$$
|\Psi\rangle=\bigotimes_{n=1}^{N}|\psi\rangle,
$$

we may also obtain an upper bound on the quantum Chernoff exponent $\xi_{\mathrm{QCB}}=-\frac{\ln Q[\Psi]}{N}$ [18]. It was shown in Ref. [18] that while the finite- $N$ behavior of $\bar{P}_{e}[\Psi]$ is complicated, $\bar{P}_{e}[\Psi]$ is asymptotically exponential in $N$ in the sense that

$$
\lim _{N \rightarrow \infty}-\frac{\ln \bar{P}_{e}[\Psi]}{N}=\xi_{\mathrm{QCB}} .
$$

Assuming $|\psi\rangle$ has finite average energy $N_{s}$ (so $|\Psi\rangle$ has average energy $N N_{s}$ ), we have from the fidelity lower bound $\mathcal{F}_{\text {min }}\left(N_{s}\right) \leqslant \mathcal{F}[\psi]$ coupled with the lower bound of (6) that

$$
\frac{1}{4}\left[\mathcal{F}_{\min }\left(N_{s}\right)\right]^{N}=\frac{1}{4}\left(r_{0} r_{1}+t_{0} t_{1}\right)^{2\left(N_{s}\right) N} \leqslant \bar{P}_{e}[\Psi]
$$

from which the bound

$$
\xi_{\mathrm{QCB}} \leqslant \ln \left[\left(r_{0} r_{1}+t_{0} t_{1}\right)^{-2 N_{s}}\right]
$$

follows on taking logarithms.

\section{Reading of an ideal memory}

For the case of $R_{1}=1$ (called "ideal memory" in Ref. [3]), the channel $\mathcal{E}_{1}$ is simply the identity channel. Therefore, the return state $\rho_{1}$ is pure: to wit, the transmitted state $|\psi\rangle\langle\psi|$. In such a situation, the fidelity $\mathcal{F}[|\psi\rangle]$ equals the Chernoff bound
$Q(|\psi\rangle)$ [18], so we have the bound [which is stronger than the upper bound of (6)]:

$$
\bar{P}_{e}[|\psi\rangle] \leqslant \frac{1}{2} \mathcal{F}[|\psi\rangle] .
$$

In conjunction with the result of Sec. IVA that the multimode Fock states attain the minimum fidelity, we have the remarkable consequence that among all the $2 M$-mode signal-idler states with signal energy $N_{s}$, a signal mode Fock state with $N_{s}$ total photons has the best (lowest) Chernoff bound. See also the discussion in Sec. IVC1.

We mention in this connection that an optimization of the Chernoff bound was carried out in Ref. [24]. However, for a fixed total energy in signal and idler, it was restricted to the cases when the input state is a single-mode squeezed thermal state and an $M=1$ signal-idler two-mode squeezed thermal state. It was shown that the single-mode and two-mode squeezed vacuum states minimize the ideal memory Chernoff bound in that class.

\section{Target detection: No-go result for large loss}

The case of target detection corresponds to $r_{0}=0$ and $t_{0}=$ 1. Under these conditions, the universal lower bound (53) reads

$$
\frac{1}{2}\left[1-\sqrt{1-\left(1-R_{1}\right)^{N_{s}}}\right] \leqslant \bar{P}_{e}[|\psi\rangle] .
$$

It is easy to check [see Eq. (61)] that the error probability obtained from a pure coherent state of energy $N_{s}$ is

$$
\bar{P}_{e \mathrm{CS}}=\frac{1}{2}\left[1-\sqrt{1-e^{-R_{1} N_{s}}}\right] .
$$

The case of large loss $R_{1} \ll 1$ is of practical importance for standoff target detection. Because $e^{-R_{1}} \simeq 1-R_{1}$ in Eq. (60) under such conditions, we see that the error probability of a general input state of energy $N_{s}$, which is lower bounded by the left-hand side of (59), is not appreciably smaller than the coherent state error probability (60). We thus have a no-go result for appreciable quantum advantage in target detection under high-loss conditions that applies to any multimode input state. Note that this does not contradict the 6-dB advantage in the error exponent of the EPR state over coherent states claimed in [2] for high-loss target detection because that analysis was carried out with the additional assumption of large thermal background noise in each signal mode.

The question arises if one can connect the fidelity with the Chernoff bound as we did in Sec. IVA2 above. If the input state has signal-idler entanglement, $R_{0}=0$ and $R_{1} \neq 1$ imply that both $\rho_{0}$ and $\rho_{1}$ are mixed in general so the argument connecting the fidelity and the Chernoff bound no longer applies. However, for the case of a pure transmitted state that is not entangled to any idler modes kept at the receiver (this is called a Type I target detection scenario in Ref. [25]), $\rho_{0}$ is a pure state, namely the vacuum state of the signal modes. Thus, it is again true that in a Type I target detection scenario, the number state transmitter yields the lowest Chernoff bound among all pure transmitted states of energy $N_{s}$. When $R_{1} \ll 1$ prevails, the no-go result given above is in force even in this case. In such regimes, the coherent state performance differs little from the number state performance [see Eq. (70) below]. This holds even for moderately large $R_{1}$, as we will see in Sec. IVE1 (see Fig. 3). 
In the remaining subsections, we compare in detail the performance of coherent states, number states, and the EPR states of Ref. [3].

\section{B. Coherent states}

For a coherent state input of energy $N_{s}$, say, the single-mode state (see Ref. [6]) $\left|\sqrt{N_{s}}\right\rangle$ of mean amplitude $\sqrt{N_{s}}$, the optimal error probability (5) for discriminating $\rho_{0}$ and $\rho_{1}$ evaluates to

$$
\bar{P}_{e \mathrm{CS}}=\frac{1}{2}\left[1-\sqrt{1-e^{-\left(r_{1}-r_{0}\right)^{2} N_{s}}}\right] .
$$

The right-hand side is exactly the lower bound on the optimal error probability of any classical state derived in Ref. [3] (Theorem 1 therein). Therefore, pure coherent states are the optimal classical states for quantum reading in the absence of added thermal noise. A physical realization of the optimal error probability (61) is provided by the so-called Dolinar receiver [26] and some other receivers exist which approximate that performance. We discuss these issues briefly in Sec. IVE2.

\section{Number states}

Let us now consider the $\bar{P}_{e}$ obtained by transmitting number states. For a transmitted Fock state $|\mathbf{N}\rangle=\left|N_{1}\right\rangle \otimes \cdots \otimes\left|N_{m}\right\rangle$ with $N_{s}=\sum_{m=1}^{M} N_{m}$, it is seen that $\left|\psi_{\mathbf{k}}^{(b)}\right\rangle=\sqrt{p_{\mathbf{k}}^{(b)}}|\mathbf{N}-\mathbf{k}\rangle$, for $p_{\mathbf{k}}^{(b)}$ a product of binomial probabilities:

$$
p_{\mathbf{k}}^{(b)}=\prod_{m=1}^{M}\left[\left(\begin{array}{c}
N_{m} \\
k_{m}
\end{array}\right) R_{b}^{N_{m}-k_{m}} T_{b}^{k_{m}}\right] .
$$

The output states $\rho_{0}$ and $\rho_{1}$ commute, so the techniques of Sec. III are not required to evaluate the performance. The optimal quantum measurement is photon counting on the individual modes followed by classical processing of the count results. It is easy to verify that the optimal decision rule is given by

$$
\frac{R_{1}^{T} \cdot T_{1}^{N_{s}-T}}{R_{0}^{T} \cdot T_{0}^{N_{s}-T}} \stackrel{\mathcal{E}_{1}}{<},
$$

for $T$ the observed total photon count (recall that we are assuming $R_{0}<R_{1}$ ). This is equivalent to the rule

$$
\begin{gathered}
\text { say } \mathcal{E}_{1} \\
T \underset{*}{\gtrless} T_{*}, \\
\text { say } \mathcal{E}_{0}
\end{gathered}
$$

where the threshold $T_{*}$ equals

$$
T_{*}=N_{s} \frac{\ln \left(\frac{T_{0}}{T_{1}}\right)}{\ln \left(\frac{R_{1} T_{0}}{R_{0} T_{1}}\right)} .
$$

Note that the decision rule is independent of the actual Fock state chosen as long as $N_{s}$ is fixed. Moreover, we can show from Eq. (62) that the probability of counting $T$ photons is also independent of the distribution of input photons among the modes as long as the total number is $N_{s}$. Consequently, $\bar{P}_{e}$ is independent of the details of the distribution of the photons

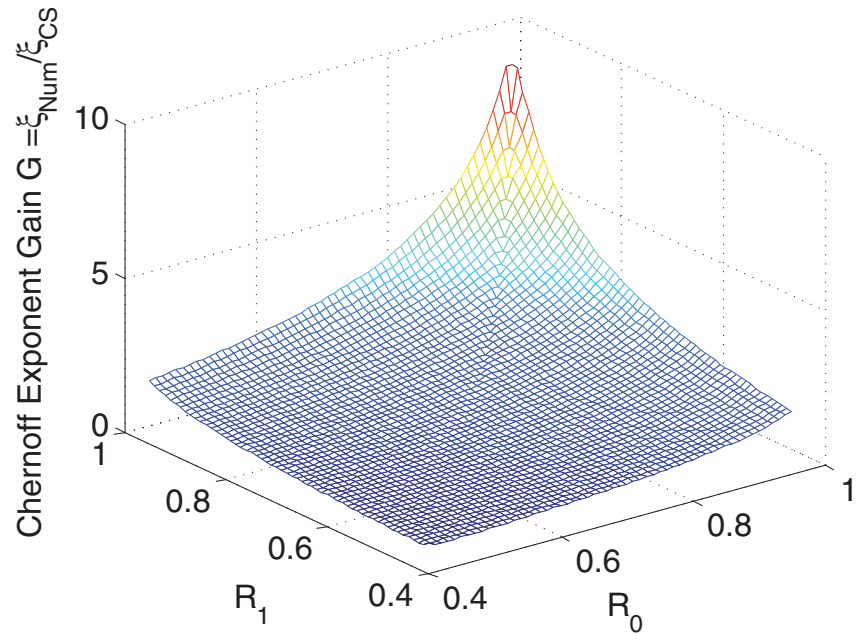

FIG. 2. (Color online) Number state vs. coherent state Chernoff exponent gain $\mathrm{G}$ of Eq. (69) for values of $R_{0}$ and $R_{1}$ above 0.4 . Appreciable gain is seen for larger values of the reflectances.

as well. This feature has practical implications that we discuss in Sec. IVE2. From Eq. (62) and inequality (63), $\bar{P}_{e}$ is easily computed numerically, as we will do in Sec. IVE1.

We also have the essentially classical Chernoff bound

$$
\bar{P}_{e} \leqslant \frac{1}{2}\left(R_{0}^{S_{*}} R_{1}^{1-s_{*}}+T_{0}^{S_{*}} T_{1}^{1-s_{*}}\right)^{N_{s}} \equiv \frac{1}{2} e^{-\xi_{\mathrm{Num}} N_{s}},
$$

where

$$
s_{*}=\left\{\ln \left[\frac{-R_{1} \ln \left(R_{0} / R_{1}\right)}{T_{1} \ln \left(T_{0} / T_{1}\right)}\right]\right\}\left\{\ln \left(\frac{T_{0} R_{1}}{T_{1} R_{0}}\right)\right\}^{-1}
$$

so $\xi_{\text {Num }}$ is the number state Chernoff exponent. This bound is obtained by computing $Q(s)$ directly from the definition (7) and finding the optimum exponent analytically. The above results apply when $R_{0} \neq 0$ and $R_{1} \neq 1$.

For a coherent state transmitter, the Chernoff bound is

$$
\bar{P}_{e} \leqslant \frac{1}{2} e^{-\left(r_{1}-r_{0}\right)^{2} N_{s}} \equiv \frac{1}{2} e^{-\xi_{\mathrm{CS}} N_{s}},
$$

for $\xi_{\mathrm{CS}}$ the coherent state Chernoff exponent. A useful measure for quantifying the improvement obtainable from a number state transmitter from coherent state performance is the ratio of their Chernoff exponents, which we call the "gain" $G$ :

$$
G=\frac{\xi_{\text {Num }}}{\xi_{\mathrm{CS}}} .
$$

In Fig. 2, we plot $G$ against the reflectances $R_{0}$ and $R_{1}$, which shows that the gain is appreciably greater than unity only when both $R_{0}$ and $R_{1}$ are fairly large. Further performance comparisons at varying values of $N_{s}$ are made in Sec. IV E.

\section{Ideal memory and target detection}

For the case $R_{0}=0$ (target detection), we have the exact result

$$
\bar{P}_{e}=\frac{1}{2} T_{1}^{N_{s}} .
$$

The optimum decision rule in this limit is to declare the target present if and only if the total count $T>0$. In the opposite limit $R_{1}=1$ (the ideal memory of Ref. [3]), we likewise obtain

$$
\bar{P}_{e}=\frac{1}{2} R_{0}^{N_{s}}
$$


and the optimum decision rule is to declare $b=1$ if and only if $T=N_{s}$. The ideal memory case is exceptional in that, irrespective of the particular (pure) state transmitted, $\rho_{1}$ is a pure state - the transmitted state itself. In such a situation, as mentioned in Sec. IVA2, the fidelity $\mathcal{F}$ equals the Chernoff bound [18]. Thus, the Chernoff bound reads

$$
\bar{P}_{e} \leqslant \frac{1}{2} \mathcal{F}
$$

which, on comparing (52) with $r_{1}=1$ to Eq. (71), is in fact an equality for Fock states.

\section{EPR state}

We now consider the $M$-mode EPR state with per-mode energy $N=N_{s} / M$ of Ref. [3], which will afford an illustration of the techniques developed in Sec. III. The EPR state is an $M$-fold tensor product of a two-mode squeezed vacuum state which, for a single mode-pair, has the photon number representation

$$
|\psi\rangle_{\mathrm{EPR}}=\sqrt{\frac{1}{N+1}} \sum_{n=0}^{\infty}\left(\frac{N}{N+1}\right)^{n / 2}|n\rangle_{I}|n\rangle_{S},
$$

where $|\psi\rangle_{\mathrm{EPR}}$ is evidently an NDS state. For the case of no added thermal noise being considered in this paper, it was shown in Ref. [3] using the Bhattacharyya bound $Q(1 / 2)$ that, for $N_{s}>N_{\text {th }}\left(R_{0}, R_{1}\right)$, a threshold total energy depending on the reflectances, there exists an $M$ for which the classical error probability is worse than the EPR Bhattacharyya bound (Theorem 2 of Ref. [3]). For the case of ideal memory, a similar threshold theorem was shown to hold with $N_{\text {th }}=1 / 2$ for all $M$ greater than a threshold $\bar{M}$ (Theorem 3 of Ref. [3]). We show here that the techniques of Sec. III may be used to derive these results in an alternative manner, as well as to provide numerical results at chosen $N$ and $M$.

We first obtain the output fidelity and $Q(s)$ for the state $|\psi\rangle_{\mathrm{EPR}}$, i.e., for $M=1$. The photon probability distribution $p_{k}^{b}$ of Eq. (27) in the output environment mode is in the BoseEinstein form of a thermal state with $T_{b} N$ average photons:

$$
p_{k}^{(b)}=\frac{1}{T_{b} N+1}\left(\frac{T_{b} N}{T_{b} N+1}\right)^{k} .
$$

Evaluating (30) yields

$$
I_{k}=\frac{\left(t_{0} t_{1} N\right)^{k}}{\left[\left(1-r_{0} r_{1}\right) N+1\right]^{k+1}} .
$$

We may now use (43) and (45) to get:

$$
\left.\mathcal{F}\right|_{M=1}=\frac{1}{\left[\left(1-r_{0} r_{1}-t_{0} t_{1}\right) N+1\right]^{2}}
$$

and

$$
\left.Q(s)\right|_{M=1}=\frac{1}{C \alpha^{s}-D \beta^{s}},
$$

with

$$
\begin{gathered}
\alpha=\frac{T_{0} N+1}{T_{1} N+1}>1, \\
\beta=\frac{T_{0}}{T_{1}}>1, \\
C=\frac{\left[\left(1-r_{0} r_{1}\right) N+1\right]^{2}}{T_{0} N+1},
\end{gathered}
$$

and

$$
D=T_{1} N
$$

We may verify that the inequalities

$$
\alpha<\beta
$$

and

$$
C>D
$$

hold [27]. For a given $N$, obtaining the Chernoff bound entails finding the exponent $s_{*}$ that minimizes (77). We know that $Q(s)$ is a convex (and continuous [28]) function of $s$ in [0,1] [18]. It is, from Eq. (77), evidently also twice differentiable in $s$. Accordingly, two cases logically arise depending on the sign of $Q^{\prime}(0)$ :

(i) If $Q^{\prime}(0) \geqslant 0, Q(s)$ is an increasing function of $s$, so $s_{*}=0$.

(ii) If $Q^{\prime}(0)<0$, then $s_{*}$ may be found by setting $Q^{\prime}\left(s_{*}\right)=$ 0 . If this equation has no solution in $[0,1], s_{*}=1$.

Differentiating Eq. (77), we find that

$$
Q^{\prime}(s)=\left(C \alpha^{s}-D \beta^{s}\right)^{-2}\left[\left(\ln \beta^{D}\right) \beta^{s}-\left(\ln \alpha^{C}\right) \alpha^{s}\right] .
$$

Consequently, the condition for deciding among the above two cases is

$$
\delta \equiv \frac{\beta^{D}}{\alpha^{C}} \underset{\text { Case 1 }}{<} 1 .
$$

In the event of Case 2, setting $Q^{\prime}\left(s_{*}\right)=0$ gives $s_{*}$ as

$$
S_{*}=\frac{\ln \left(\frac{\ln \alpha^{C}}{\ln \beta^{D}}\right)}{\ln \left(\frac{\beta}{\alpha}\right)} .
$$

In the event that the right-hand side of (86) is greater than 1 , we have $s_{*}=1$.

Finally, from the multiplicative properties of the fidelity and the Chernoff-type quantities, we have that, for the $M$-mode input state $|\psi\rangle=\otimes_{m=1}^{M}|\psi\rangle_{\mathrm{EPR}}$, the fidelity equals

$$
\mathcal{F}_{\mathrm{EPR}}=\left[\left(1-r_{0} r_{1}-t_{0} t_{1}\right) N+1\right]^{-2 M}
$$

and the Chernoff bound is

$$
\bar{P}_{e} \leqslant \frac{1}{2} Q_{\mathrm{EPR}}=\frac{1}{2} Q\left(s_{*}\right)=\frac{1}{2}\left[C \alpha^{s_{*}}-D \beta^{s_{*}}\right]^{-M}
$$

for the $s_{*}$ obtained from the above case analysis.

When the input state $|\psi\rangle$ is a multimode Gaussian state, i.e., a state whose Wigner function is a Gaussian probability 
distribution (see, e.g., Ref. [29]), so are $\rho_{0}$ and $\rho_{1}$ since $\mathcal{E}_{b}^{\otimes^{M}} \otimes$ id $^{\otimes^{M^{\prime}}}$ is a linear, and hence Gaussian, channel. The input state $|\psi\rangle_{\mathrm{EPR}}$ is a Gaussian state. We could, therefore, use the general Gaussian state technique of Ref. [30] to derive the fidelity between $\rho_{0}$ and $\rho_{1}$. Similarly, the technique of symplectic diagonalization [19] may be used to derive $Q(s)$, as it was in Ref. [3].

Let us now connect these results to Theorems 2 and 3 of Ref. [3]; the statements of these theorems were reviewed in the first paragraph of this subsection. Theorem 2 makes essential use of the large $M$ limit of the Bhattacharyya bound $Q(1 / 2)$ given by (7). Some lengthy but straightforward algebra verifies that the $M \rightarrow \infty$ limit (at constant $N_{s}=M N$ ) of the $M$-pair Bhattacharyya bound $B\left(N_{s}\right)$

$$
B\left(N_{S}\right)=\frac{1}{2} \lim _{M \rightarrow \infty}\left[\left.Q(1 / 2)\right|_{M=1}\right]^{M}
$$

for $\left.Q(1 / 2)\right|_{M=1}$ obtained from Eq. (77) is identical to the corresponding result (117) of Ref. [31] after adjusting for different notation (see Ref. [32]). The same reasoning in Ref. [31] that establishes the "threshold energy" theorem (Theorem 2 of Ref. [3]) may then be carried out from Eq. (89) to give an identical threshold energy as before.

Consider now the case of an ideal memory with $T_{1}=0$ for comparison to Theorem 3 of Ref. [3]. That theorem depends on the expression (129) of Ref. [31] for the Chernoff bound which we rederive using the method of this section. The same analysis as in Ref. [31] can then be used to reproduce the result of Theorem 3. From Eq. (81), we have $D=0$ so Eq. (77) is clearly minimized at $s_{*}=1$ so the $M$-mode Chernoff bound is

$$
\bar{P}_{e} \leqslant \frac{1}{2} Q_{\text {ideal }}=\frac{1}{2}\left[\left(1-r_{0}\right) N+1\right]^{-2 M}
$$

which agrees with (129) of Refs. [31,32]. Further, we see that the fidelity (87) also equals $Q_{\text {ideal }}$ as it should because $\rho_{1}$ is a pure state. In the general case of $R_{1} \neq 1$, we can study the behavior of the Chernoff bound as a function of $M$ and $N_{s}$ by numerically obtaining $s_{*}$ for each value of these parameters.

\section{E. Comparison of coherent state, number state, and EPR state transmitters}

\section{Numerical comparison of error probability}

In this subsection, we compare quantitatively the error probability performance of the three types of states considered in the previous subsections for target detection and reading of nonideal and ideal memories. The representative plots below show the error probability on the $y$ axis in logarithmic scale against the total average signal energy $N_{s}$ on the $x$ axis. We assume the number of modes $M=50$. We reiterate that the number of modes has no effect on either the coherent state or number state performance, which depend on $N_{s}$ alone. For the EPR state, varying $M$ changes the performance (as given by the Chernoff bound), although the change is not appreciable once $M$ is around 20-30. Thus, the plots given here are fairly representative of the best possible EPR state performance.

We summarize how the plots were made. For each $N_{s}$, the universal lower bound of (53) was plotted in Figs. 2-5. The coherent state error probability is given by the closed

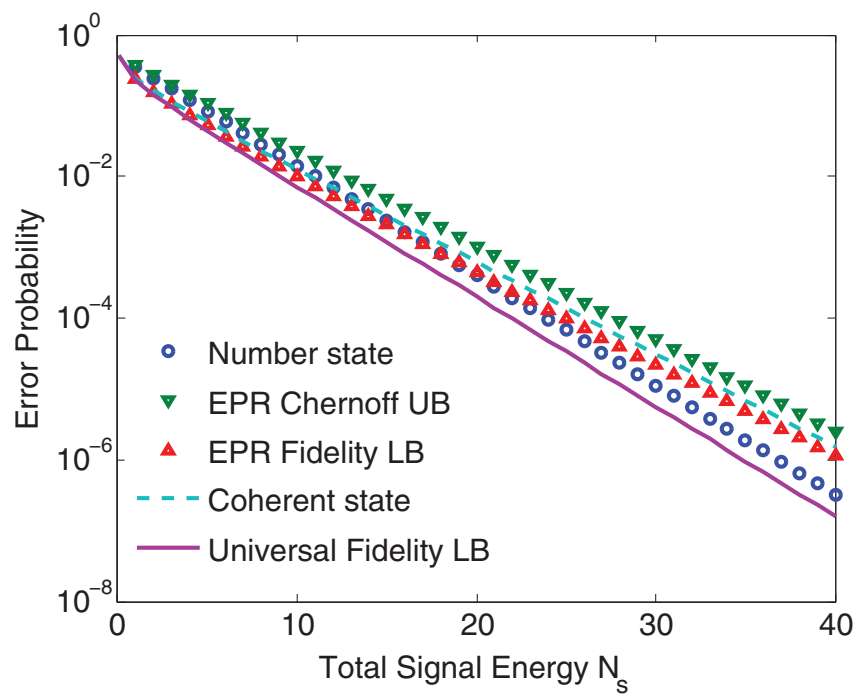

FIG. 3. (Color online) Error probability bounds versus $N_{s}$ for target detection with $R_{0}=0$ and $R_{1}=0.3$. The number of modes $M=50$ for the EPR state curves.

form expression (61). The number state error probability was calculated numerically for each value of $N_{s}$ using the count probability distribution (62) and the decision rule (63) for the cases of reading of nonideal memories. The number state Chernoff bound (66)-(67) was also plotted for these cases. Closed-form expressions (70) and (71) were used for the detection and ideal memory cases. The EPR state fidelity expression (87) in conjunction with (6) gives the EPR fidelity lower bound

$$
\frac{1}{2}\left(1-\sqrt{1-\mathcal{F}_{\mathrm{EPR}}}\right) \leqslant \bar{P}_{e \mathrm{EPR}} .
$$

Finally, for each value of $N_{S}$, the EPR Chernoff bound (88) was calculated for the detection and nonideal memory cases using the procedure for obtaining $s_{*}$ described in Sec. IV D.

In all the figures, we see that the number state and EPR state transmitters eventually outperform the coherent state transmitter, in tune with the conclusions of Ref. [3]. In the target detection case (Fig. 3), the number state also outperforms the EPR state lower bound, although the performance difference between the three states is not appreciable. We see that, even for an $R_{1}$ that is much larger than that expected in a realistic target detection scenario, the coherent state performance is not appreciably worse than the number state performance. As $R_{0}$ increases, the perfomance gain over classical increases also as evidenced in Figs. 4-7. Figure 4 shows a case where the difference $R_{1}-R_{0}$ is small and the reflectances themselves are not very high. In such cases, the number state and coherent state performances do not differ appreciably. However, the EPR Chernoff bound drops below the number state performance for $N_{s}$ greater than about 40 photons. Figure 5 also shows a case of small $R_{1}-R_{0}$, but the reflectances themselves are appreciable. We see that the nonclassical transmitters' gain over the coherent state increases. Further, the crossover between the number state performance and the EPR Chernoff bound occurs later (at about 65 photons) and the slopes of these two curves different less than in Fig. 4. Figure 6 represents distinguishing channels with large $R_{1}-R_{0}$. We see that the 


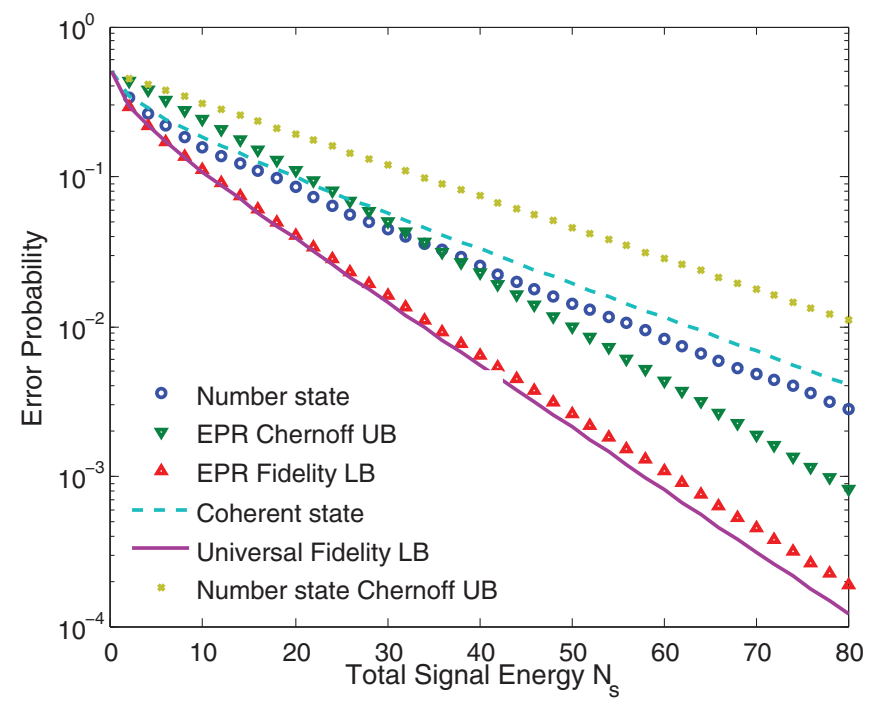

FIG. 4. (Color online) Error probability bounds versus $N_{s}$ for quantum reading with $R_{0}=0.3$ and $R_{1}=0.6$. The number of modes $M=50$ for the EPR state curves.

nonclassical gain over the coherent state transmitter is even greater, with the EPR state doing better than the number state; the crossover of the number state performance with the EPR Chernoff bound now occurs at about 20 photons. Finally, Fig. 7 represents the reading of an ideal memory with $R_{0}=0.5$. The nonclassical gain is now very large, and the number state transmitter performs better than the EPR state, as evinced by the fact that it lies below the EPR state lower bound. All the plots are consistent with the number state versus coherent state Chernoff exponent gain of Fig. 2, and Fig. 7 confirms and strengthens the conclusion of Section IVA2 that the number state transmitter has the lowest Chernoff bound for reading of an ideal memory.

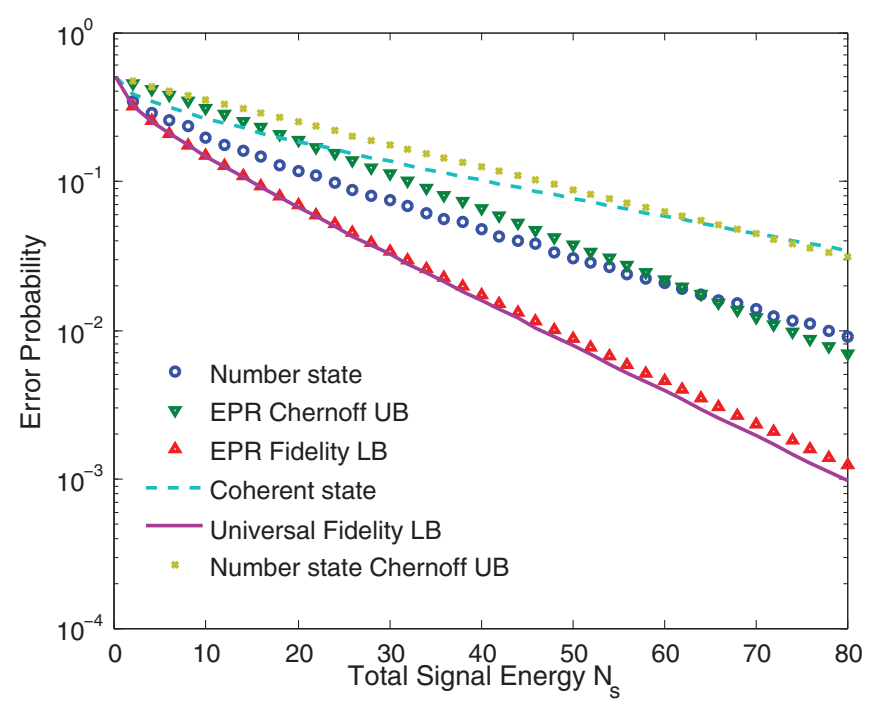

FIG. 5. (Color online) Error probability bounds versus $N_{s}$ for quantum reading with $R_{0}=0.5$ and $R_{1}=0.75$. The number of modes $M=50$ for the EPR state curves.

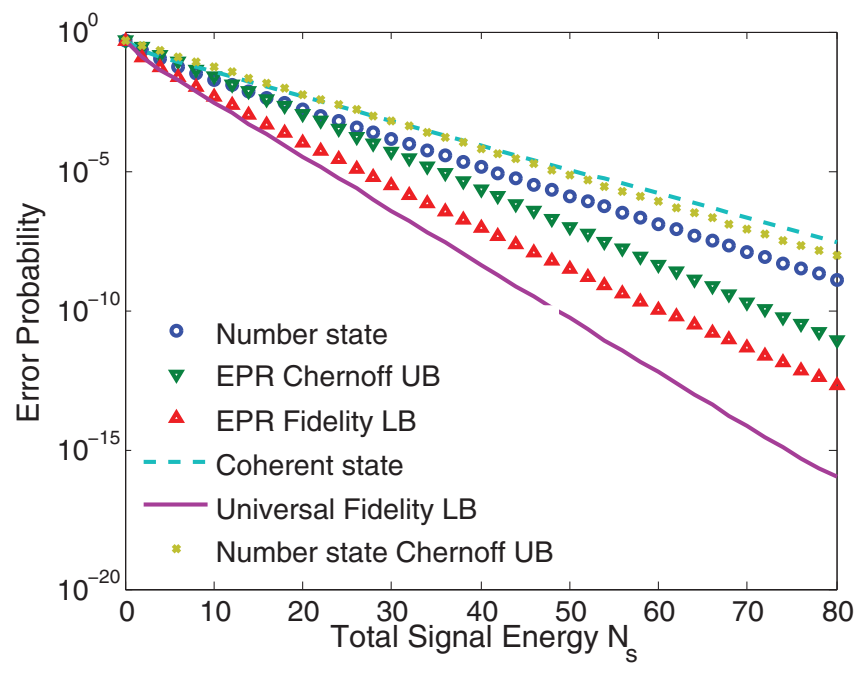

FIG. 6. (Color online) Error probability bounds versus $N_{s}$ for quantum reading with $R_{0}=0.2$ and $R_{1}=0.8$. The number of modes $M=50$ for the EPR state curves.

\section{Experimental considerations}

In this section, we indicate some technological considerations regarding the availability of sources and detectors that bear on the implementation of quantum reading with coherent, number, and EPR states.

While coherent sources of any energy are readily available, the optimal Helstrom detector of Ref. [26] is not easy to realize as it employs feedback in addition to linear optics and photodetection. Nevertheless, the Kennedy receiver [33] and the so-called optimum displacement receiver (ODR) [34] achieve the same error exponent and are more easily implemented since they do not involve feedback. Indeed, the ODR was recently demonstrated [35] with an overall detection efficiency of $\sim 90 \%$.

A single-mode number state with $N_{s}>1$ is hard to generate with current technology. However, we saw in Sec. IVC that

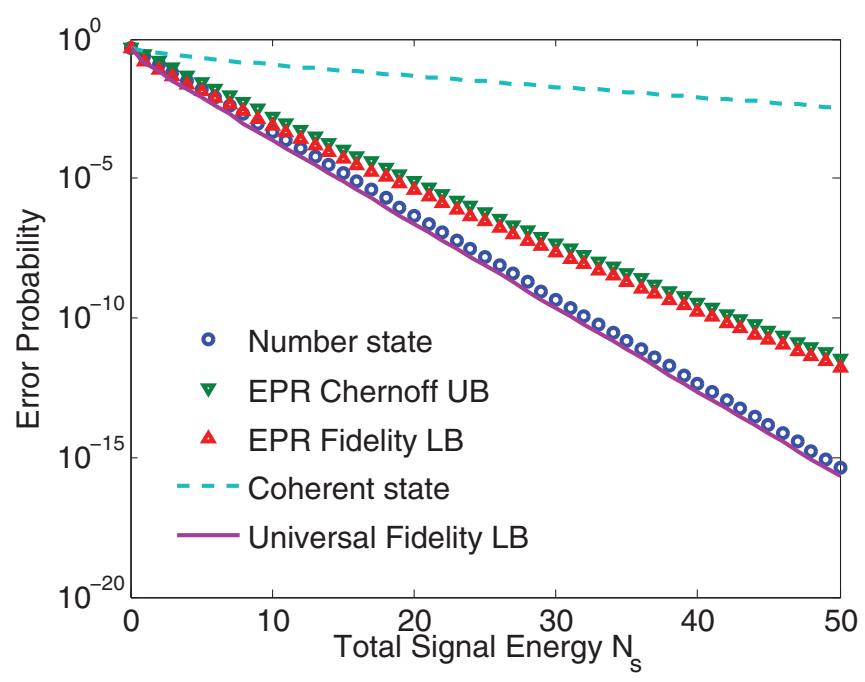

FIG. 7. (Color online) Error probability bounds versus $N_{s}$ for reading of an ideal memory with $R_{0}=0.5$ and $R_{1}=1$. The number of modes $M=50$ for the EPR state curves. 
a state consisting of $N_{s}$ spatial or temporal modes, each of which is in a single photon state, i.e., a state of the form $|1\rangle \otimes \cdots \otimes|1\rangle$ with $N_{s}$ total photons, has identical performance. As a large variety of single-photon sources are available (see, e.g., Ref. [36]), the generation of multimode Fock state with many modes each in a single photon state does not appear problematic with current technology. The optimal Helstrom measurement is photon counting on the individual modes followed by classical processing. While high-quantum-efficiency photon number-resolving detectors are still a developing technology (see, e.g., Ref. [37]) and require cooling to superconducting temperatures, the source described above consisting of multiple single-photon states requires only standard single-photon avalanche diode (SPAD) [38] technology with either one or many detectors depending on the mode implementation. Detector quantum efficiencies and other system losses act as multiplicative factors to $R_{0}$ and $R_{1}$ and do not essentially change the analysis described in Sec. IVC, as also is the case for the coherent state transmitter result of Sec. IVB. SPADs, for instance, can attain quantum efficiencies of around $\eta \sim 0.75$. After adjusting $R_{0}$ and $R_{1}$ to account for system inefficiencies, it is fair to say that the optimum Helstrom receiver for quantum reading with number states is realizable with current technology.

Generating multimode EPR states of high total energy $N_{s}$ and large $M$ also does not seem to present a huge experimental problem (see, e.g., Ref. [39]). Unfortunately, the optimum Helstrom detector, while given abstractly by Eqs. (40) and (41), is an entangling measurement over the $M$ modes and has no known concrete realization. A realizable suboptimal measurement involving homodyne detection was proposed in Refs. [3,31] and was shown, significantly, to also outperform the coherent state transmitter. However, it appears that this comparison was made in Ref. [31] only for rather high $R_{0}$ and $R_{1}$. The effects of nonunity homodyne detector quantum efficiency, analogously to the Fock state and coherent state cases above, have also not yet been considered in that measurement. Moreover, given the comparisons made in this paper, it is of interest to see how the suboptimal measurement compares to the number state performance for reasonable values of $N_{s}$ and $M$.

\section{CONCLUSION}

The problem of distinguishing two optical beam-splitter channels using multimode signal-idler entangled pure states was considered. A general lower bound on the output state fidelity and minimum error probability for any such input was derived. For NDS states, series formulas for the optimum error probability, the output state fidelity, and the Chernofftype upper bounds were derived. For quantum reading and target detection, for a given signal photon probability mass function, the fidelity bound was shown to be attained by NDS states, with multimode Fock states minimizing the bound for a given total photon number. For reading of an ideal memory with arbitrary states and for Type I (signal-only) target detection, the number state was shown to yield the best Chernoff bound among all states of given energy. For target detection under high-loss conditions, a general no-go result for quantum advantage over coherent states was obtained. The above results were applied to quantitatively studying the performance gains over classical states obtainable by number state and EPR state transmitters, which were found to outperform the classical transmitters to varying degrees over a wide range of reflectances. The experimental outlook on realizing the optimal measurement for the number state transmitter was argued to be good. It is of interest to compare the performance, taking into account realistic experimental parameters, of the non-Helstrom measurement on the EPR state transmitter suggested in Ref. [3] and the number state transmitter suggested here. Finally, the techniques developed here are likely to prove useful for other interesting problems fitting the same framework, e.g., the lossy discrimination of optical phase shift channels.

\section{ACKNOWLEDGMENTS}

The author is grateful to Alessandro Bisio, Michele Dall'Arno, Giacomo M. D'Ariano, Saikat Guha, Bhaskar Mookerji, Stefano Pirandola, Jeffrey H. Shapiro, Franco N. C. Wong, Brent J. Yen, and Horace P. Yuen for useful discussions. This material is based on work funded by DARPA's Quantum Sensor Program under AFRL Contract No. FA8750-09-C0194.
[1] C. Helstrom, Quantum Detection and Estimation Theory (Academic Press, New York, 1976).

[2] S.-H. Tan, B. I. Erkmen, V. Giovannetti, S. Guha, S. Lloyd, L. Maccone, S. Pirandola, and J. H. Shapiro, Phys. Rev. Lett. 101, 253601 (2008).

[3] S. Pirandola, Phys. Rev. Lett. 106, 090504 (2011).

[4] E. C. G. Sudarshan, Phys. Rev. Lett. 10, 277 (1963).

[5] R. J. Glauber, Phys. Rev. 131, 2766 (1963).

[6] It is enough to consider this essentially single-mode coherent state since its output under $\mathcal{E}_{\mathrm{b}}^{\otimes^{M}}$ is readily converted through an $M$-mode beam-splitter transformation to the output under the same channel of any other product coherent state of the same total energy.

[7] A. Bisio, M. Dall'Arno, and G. M. D'Ariano, Phys. Rev. A 84, 012310 (2011).
[8] A. Uhlmann, Rep. Math. Phys. 9, 273 (1976).

[9] R. Jozsa, J. Mod. Opt. 41, 2315 (1994).

[10] We note that the definition (4) of fidelity agrees with that in Refs. [8,9] but is the square of the definition in Ref. [21].

[11] R. Nair and B. J. Yen, e-print arxiv:1107.1190v2 (2011).

[12] V. C. Usenko and M. G. A. Paris, Phys. Rev. A 75, 043812 (2007)

[13] J. P. Dowling, Contemp. Phys. 49, 125 (2008).

[14] S. D. Huver, C. F. Wildfeuer, and J. P. Dowling, Phys. Rev. A 78, 063828 (2008).

[15] G. S. Agarwal, Phys. Rev. Lett. 57, 827 (1986).

[16] T.-W. Lee, S. D. Huver, H. Lee, L. Kaplan, S. B. McCracken, C. Min, D. B. Uskov, C. F. Wildfeuer, G. Veronis, and J. P. Dowling, Phys. Rev. A 80, 063803 (2009). 
[17] C. A. Fuchs and J. van de Graaf, IEEE Trans. Inf. Theory 45, 1216 (1999).

[18] K. M. R. Audenaert, J. Calsamiglia, R. Munoz-Tapia, E. Bagan, L. Masanes, A. Acin, and F. Verstraete, Phys. Rev. Lett. 98, 160501 (2007).

[19] S. Pirandola and S. Lloyd, Phys. Rev. A 78, 012331 (2008).

[20] While the addition of effectively thermal noise from decoherence effects and by stray photons of the order of $10^{-5}$ photons per mode was also considered in Ref. [3], we do not do so here because it appears difficult to carry over the analysis presented in this paper to the more general case of added thermal noise.

[21] M. A. Nielsen and I. L. Chuang, Quantum Information and Computation (Cambridge University Press, New York, 2000).

[22] Equations (26) and (29) together imply by the Cauchy-Schwarz inequality that $I_{\mathbf{k}} \leqslant \sqrt{p_{\mathbf{k}}^{(0)} p_{\mathbf{k}}^{(1)}}$ so the expression under the square root in Eqs. (36) and (39) is non-negative.

[23] G. H. Hardy, J. E. Littlewood, and G. Pólya, Inequalities, 2nd ed. (Cambridge University Press, New York, 1988).

[24] C. Invernizzi, M. G. A. Paris, and S. Pirandola, e-print arXiv:1011.2785v5 (2010).

[25] H. P. Yuen and R. Nair, Phys. Rev. A 80, 023816 (2009).

[26] S. J. Dolinar, MIT Research Laboratory of Electronics Technicla Report 111, 1973.

[27] From Eqs. (80) and (81), $C>D$ can be shown to be equivalent to a quadratic polynomial in $N$ with coefficients which are functions of the beam-splitter parameters being positive. Using $R_{0}<R_{1}$ along with the arithmetic mean-geometric mean inequality, each of the coefficients may be shown to be positive, yielding the result.
[28] Indeed, the smallest value taken by the denominator of (77) for $s \in[0,1]$ is lower bounded by $C-D \beta$ which can be shown to be positive. Hence, $Q(s)$ remains bounded in $[0,1]$.

[29] S. L. Braunstein and P. van Loock, Rev. Mod. Phys. 77, 513 (2005).

[30] Gh.-S. Paraoanu and H. Scutaru, Phys. Rev. A 61, 022306 (2000).

[31] S. Pirandola, Phys. Rev. Lett. 106, 090504 (2011), [online supplementary material]

[32] In keeping with usual optics notation, we have used $R_{\mathrm{b}}$ in lieu of $r_{\mathrm{b}}$ of Ref. [3] for the beam-splitter (energy) reflectances, reserving the lower case symbols for the magnitudes of field reflection coefficients. We use $N_{s}$ for the average total signal energy throughout this paper which corresponds to $N$ of Ref. [3]. We use $N$ in Sec. IVD for the per-mode EPR state energy so our $N=N_{s} / M$.

[33] R. S. Kennedy, MIT Research Laboratory of Electronics Technical Report 108, 1973.

[34] M. Takeoka and M. Sasaki, Phys. Rev. A 78, 022320 (2008).

[35] K. Tsujino, D. Fukuda, G. Fujii, S. Inoue, M. Fujiwara, M. Takeoka, and M. Sasaki, Phys. Rev. Lett. 106, 250503 (2011).

[36] B. Lounis and M. Orrit, Rep. Prog. Phys. 68, 1129 (2005).

[37] D. Rosenberg, A. E. Lita, A. J. Miller, and S. W. Nam, Phys. Rev. A 71, 061803(R) (2005).

[38] B. E. A. Saleh and M. C. Teich, Fundamentals of Photonics, 2nd ed. (John Wiley \& Sons, New York, 2007).

[39] F. N. C. Wong, J. H. Shapiro, and T. Kim, Laser Phys. 16, 1517 (2006). 